\title{
Soluble Suppression of Tumorigenicity-2 Levels as Prognostic Marker in Non-ST-Segment Elevation Myocardial Infarction
}

\author{
Sherly Purnamawaty, Tenri Esa, Ibrahim Abd Samad
}

Department of Clinical Pathology, Faculty of Medicine, Hasanuddin University/Dr.Wahidin Sudirohusodo Hospital, Makassar, Indonesia. E-mail: sherlypurnamawaty@gmail.com

\begin{abstract}
Acute Myocardial Infarction (IMA) is the most severe manifestation of coronary arterial disease, and about $60 \%-75 \%$ of IMA is NSTEMI. It is known that complications are associated with high mortality rates; therefore, predicting the development of complications in NSTEMI will help physicians improve risk stratification and determine optimal treatment. Suppression of tumorigenicity-2 (ST2) is a family of interleukin-1 (IL-1) receptors. Ischemia, injury, and myocardial infarction will cause cardiomyocytes to release SST2 associated with a worse prognosis. This study aimed to analyze sST2 levels in NSTEMI patients as a prognostic marker. This study used a prospective cohort method performed on NSTEMI patients treated at Pusat Jantung Terpadu of Dr. Wahidin Sudirohusodo Hospital during March 2019. Forty-two patients were involved as samples. All patients were tested for SST2 levels by immunochromatography and followed up during hospitalization. Data on the development of heart failure, arrhythmia, cardiogenic shock, sudden cardiac arrest, length of stay, and outcome were recorded during follow-up. Data were statistically analyzed with Mann-Whitney and Spearman test. The results of the SST2 level in NSTEMI with and without heart failure were $114.09 \pm 92.01 \mathrm{ng} / \mathrm{mL}$ and $58.94 \pm 57.75 \mathrm{ng} / \mathrm{mL}$ $(p=0.014)$, respectively. There was no significant difference between sST2 levels in NSTEMI with complications of arrhythmias, cardiogenic shock, and sudden cardiac arrest compared and patients without those complications ( $p>0.05$ ). The level of sST2 was significantly higher in NSTEMI patients who passed away $(164.05 \pm 77.35 \mathrm{ng} / \mathrm{mL})$ than those who survived $(72.55 \pm 73.15(p=0.027)$. There was no correlation between $s S T 2$ levels and length of stay ( $p=0.947)$. It was concluded that SST2 levels could be a prognostic marker for NSTEMI, particularly heart failure and outcome.
\end{abstract}

Keywords: NSTEMI, sST2 heart failure, arrhythmia, cardiogenic shock, sudden cardiac arrest, mortality

\section{INTRODUCTION}

Acute Myocardial Infarction (AMI) is the most severe manifestation of coronary artery disease as a result of cardiomyocytes death that occurs due to a lack of oxygen supply. There were 2.4 million deaths in the United States, more than 4 million deaths in Europe, and more than one third of all causes of death in developed countries every year. ${ }^{1}$ According to World Health Organization data, mortality due to heart disease in Indonesia is 191-541 people per 100,000 male population and 112-334 people per 100,000 female population. ${ }^{2}$ The development of therapy and changes in lifestyle decreases the incidence of ST-Segment Elevation Myocardial Infarct (STEMI), leading to the increase of the proportion of Non-ST-Segment Elevation Myocardial Infarct (NSTEMI) each year. About $60 \%-75 \%$ of AMI is currently NSTEMI. ${ }^{1}$

The most common complication of AMI, both STEMI, and NSTEMI, is heart failure. Other complications that can occur are cardiogenic shock, arrhythmia, and sudden cardiac arrest. ${ }^{3,4}$ The incidence of heart failure in STEMI was similar to NSTEMI in AMI patients (Global Registry of Acute Coronary Events/GRACE). ${ }^{5}$ The NSTEMI mortality rate during hospitalization and during follow-up has dropped dramatically due to advanced treatment; however, short-term and long-term complications remain to be associated with high mortality. Predicting complications in NSTEMI patients will help doctors improve risk stratification and to determine optimal treatment plans. ${ }^{6}$ Biomarkers can help in risk stratification and can be a guide to optimize the treatment plans. Research on biomarkers for cardiac fibrosis and remodeling such as galectin-3 and SST2 are currently being developed?

Suppression of tumorigenicity 2 (ST2) is a family of interleukin-1 (IL-1) receptors. ST2 isoforms are classified into transmembrane (ST2L) and soluble forms (sST2). ST2 is a receptor for interleukin-33 (IL-33), which is secreted by cardiomyocytes in response to cell damage. Interleukin-33 has an effect 
by binding to the ST2L isoform. The interaction between IL-33 and ST2L has been shown to be cardioprotective. ${ }^{8}$ The soluble form of ST2 binds strongly to IL-33 and competes with ST2L. The interaction between soluble receptors and IL-33 will inhibit the bond between IL-33 and ST2L. It results in the loss of cardioprotective effects of $\mathrm{IL}-33 / \mathrm{ST} 2 \mathrm{~L}$, leading to progressive changes in the ventricles that are consistent with remodeling, cardiac fibrosis, progressive heart failure, and death. ${ }^{9}$ Progressive cardiac fibrosis is a significant factor that plays a role in the progression of cardiac dysfunction and is also a major cause of lethal arrhythmias and sudden cardiac death. ${ }^{10}$

Myocardial ischemia, injury, and infarction will cause SST2 release by cardiomyocytes. Research on SST2 levels in AMI showed that SST2 could be a predictor of cardiovascular mortality and was useful in risk stratification of AMI. ${ }^{11}$ SST2 levels were found to be elevated in NSTEMI and related to one-year mortality. ${ }^{12}$ Research conducted in STEMI patients showed that SST2 levels had excellent sensitivity for prognosis during hospitalization, especially in the development of arrhythmias, heart failure, and reinfarction. ${ }^{13}$ A study analyzing SST2 levels in myocardial infarction in association with adverse cardiac events (including arrhythmias, cardiogenic shock, heart failure, and sudden cardiac arrest) reported that elevated SST2 levels could be a predictor of adverse cardiac events. ${ }^{14}$ Based on the background, this study aimed to analyze SST2 levels in NSTEMI patients as a prognostic marker.

\section{METHODS}

This research was a prospective cohort study performed in Dr. Wahidin Sudirohusodo Hospital Makassar from March to April 2019. The ethical committee of Health Research of Hasanuddin University approved the study protocol No.159/UN4.6.4.5.31/PP36/2019. All patients diagnosed with NSTEMI were recruited as study subjects. Diagnosis of NSTEMI was determined by a cardiologist based on anamnesis, physical examination, electrocardiogram, and cardiac markers. Patients with rheumatoid arthritis, autoimmune diseases, malignancy, sepsis, and liver failure were excluded. Serum samples were obtained by venipuncture of the subjects on admission. All the samples were tested for the SST2 levels in Prodia Laboratory. The test used sandwich monoclonal lateral flow immunoassay methods, and the results were reported in $\mathrm{ng} / \mathrm{mL}$. During hospitalization, the subjects were then followed-up for the development of heart failure, cardiogenic shock, arrhythmia, and sudden cardiac arrest diagnosed by a cardiologist. The endpoint of the follow-up was the death or discharge of patients. The outcome (death or discharge) and length of stay data were also recorded.

Statistical analysis was performed using SPSS. Mann-Whitney $U$ test was used to compare the SST2 levels between the groups with and without complications and between the death and survival groups. Spearman correlation test was used to determine the correlation between SST2 level and length of stay. P-value $<0.05$ was considered as statistically significant.

\section{RESULTS AND DISCUSSION}

A total of 42 subjects with NSTEMI met the inclusion criteria. Subjects aged between $34-80$ years with a mean of 57.4 years. $28(66.7 \%)$ male subjects were predominantly found compared to females. Risk factors for Coronary Heart Disease (CHD) such as hypertension (40.5\%), diabetes mellitus (35.7\%), smoking (40.5\%), history of CHD (42.9\%), and dyslipidemia $(76.2 \%)$ were found in this study. The average sST2 levels was $81.26 \mathrm{ng} / \mathrm{mL}$ with a range between $<12.5->250 \mathrm{ng} / \mathrm{mL}$. Subjects characteristics are summarized in Table 1.

Epidemiological data show that the incidence of myocardial infarction, including NSTEMI is higher in males than female, and the risk increases with age. . $^{15,16}$ This study was consistent with the study by Amarendra et al., which found that the majority of NSTEMI subjects were male (68\%) and the average age was 60.9 years. Old age and male are risk factors associated with atherosclerosis and $\mathrm{CHD} .^{17}$

Another study on African American NSTEMI patients found the highest prevalence of hypertension risk factors (91\%) smoking (70\%), dyslipidemia (36\%), and diabetes mellitus (25\%). ${ }^{18}$ In addition to unmodifiable risk factors such as age, male, genetic predisposition, there are still many other risk factors that can be modified by lifestyle changes and/or pharmacotherapy. Important risk factors include dyslipidemia, hypertension, diabetes mellitus, smoking, and obesity. ${ }^{19}$

Table 2 shows the differences in SST2 levels in complications of NSTEMI. Mann Whitney test was used to analyze the differences in SST2 levels in each group who had complications of heart failure, cardiogenic shock, arrhythmia, and sudden cardiac arrest compared to the group of patients without complications. SST2 levels in patients with heart failure were significantly higher than SST2 levels in 
Table 1. Subjects characteristics $(n=42)$

\begin{tabular}{|c|c|c|c|}
\hline Variable & n (\%) & Mean $\pm S D$ & Median (min-max) \\
\hline Age (year) & & $57.4 \pm 9.6$ & $56.5(34-80)$ \\
\hline \multicolumn{4}{|l|}{ Gender } \\
\hline Male & $28(66.7 \%)$ & & \\
\hline Female & $14(33.3 \%)$ & & \\
\hline \multicolumn{4}{|l|}{ Risk factors } \\
\hline Hypertension & $28(66.7 \%)$ & & \\
\hline Diabetes mellitus & $15(35.7 \%)$ & & \\
\hline Smoking & $17(40.5 \%)$ & & \\
\hline CHD history & $18(42.9 \%)$ & & \\
\hline Dyslipidemia & $32(76.2 \%)$ & & \\
\hline \multicolumn{4}{|l|}{ Symptom onset } \\
\hline$<12$ hours & 21 (50\%) & & \\
\hline$\geq 12$ hours & 21 (50\%) & & \\
\hline sST2 (ng/mL) & & $81.26 \pm 77.49$ & $47.60(12.5-250)$ \\
\hline$<35$ & 17 (40.5) & & \\
\hline$\geq 35$ & $25(59.5 \%)$ & & \\
\hline \multicolumn{4}{|l|}{ Complications } \\
\hline Heart failure & $17(40.5 \%)$ & & \\
\hline Cardiogenic shock & $3(7.1 \%)$ & & \\
\hline Arrhythmia & 9 (21.4\%) & & \\
\hline Sudden cardiac arrest & $2(4.8 \%)$ & & \\
\hline Length of stay (day) & & $7.9 \pm 4.4$ & $7(2-23)$ \\
\hline Deaths & $4(9.5 \%)$ & & \\
\hline
\end{tabular}

Table 2. Differences of sST2 levels in complications of NSTEMI

\begin{tabular}{lccc}
\hline & \multicolumn{2}{c}{$\mathbf{s S T 2}(\mathbf{n g} / \mathbf{m L})$} & \multirow{2}{*}{$\mathbf{p}^{*}$} \\
\cline { 2 - 3 } & Mean & SD & 0.014 \\
Heart failure & 114.09 & 92.01 & \\
Yes & 58.94 & 57.75 & 0.927 \\
No & & \\
Arrhythmia & 84.86 & 87.07 & 0.558 \\
Yes & 80.28 & 76.13 & \\
No & 107.63 & 79.23 & 0.595 \\
Cardiogenic shock & 79.23 & 74.95 & \\
Yes & & & \\
No & 109.8 & 115.68 & \\
Sudden cardiac arrest & 79.83 & 76.99 & \\
Yes & & \\
No & & & \\
\hline
\end{tabular}

patients without heart failure $(p=0.014)$, as shown in Figure 1.

Another large-scale study with a total of 4,426 NSTEMI patients showed high levels of sST2 associated with the incidence of heart failure in both early and late-onset. ${ }^{20}$ Research showed that when the ST2 gene was inactivated, and large amounts of SST2 were given, progressive changes in the ventricles consistent with remodeling, progressive heart failure, and death were observed. ${ }^{21}$ IL-33/ST2L cardioprotective effects occur through inhibition of signaling pathways of nuclear factor kappa light chain enhancer of activated B cells (NF-KB), extracellular response kinase 1 (ERK-1) and protein activator 1 (AP-1) which play animportant role in remodeling. ${ }^{22}$ The anti-hypertrophic effect of IL-33 occurs through inhibition of the effects of angiotensin II, which causes cardiac hypertrophy by inducing NF-kB in the 


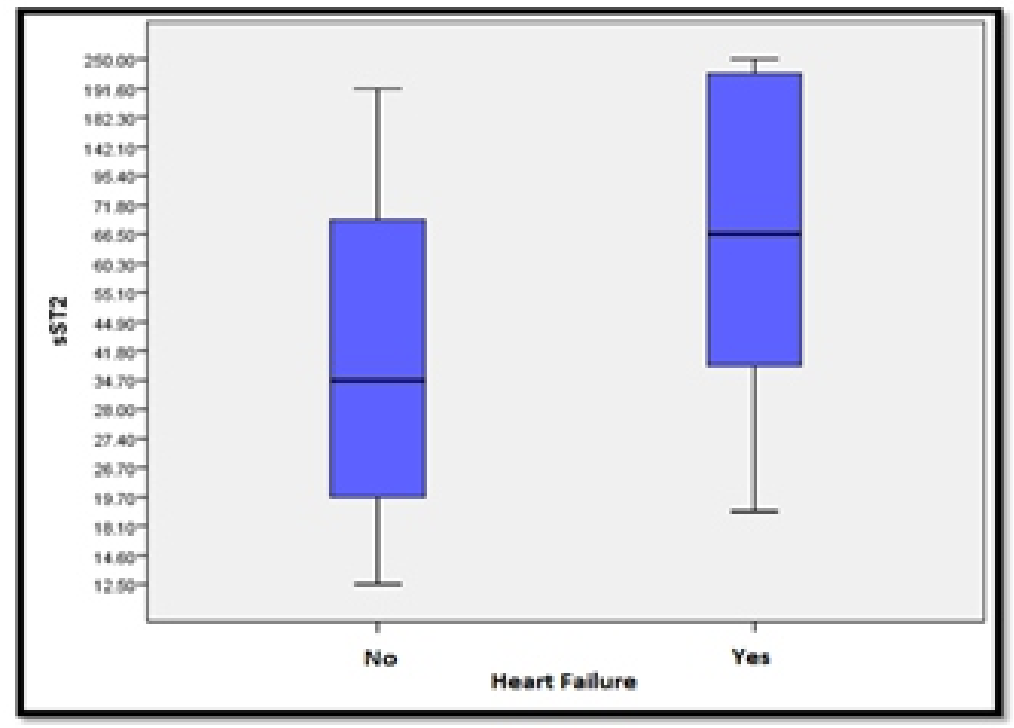

Figure 1. The difference in SST2 levels between groups with and without heart failure

myocardium. Increased sST2 levels will cause the loss of antiremodeling and anti-hypertrophic effects which result in heart failure. ${ }^{23}$

SST2 levels were higher in the group of patients with arrhythmias compared to the group without arrhythmias, even though it was not statistically significant $(p=0.927)$. Similar results were also obtained when comparing groups of patients with and without cardiogenic shock $(p=0.558)$ and between groups with and without sudden cardiac arrest $(p=0.595)$. Several studies have analyzed SST2 levels in myocardial infarction by correlating SST2 levels with adverse cardiac events (arrhythmias, cardiogenic shock, heart failure, and sudden cardiac arrest) and reported that elevated SST2 levels could be a predictor of adverse cardiac events. ${ }^{13,14}$ Progressive cardiac fibrosis is a significant factor that plays a role in the progression of cardiac dysfunction and is also a major cause of lethal arrhythmias and sudden cardiac death. Cardiac fibrosis markers, including SST2 should be related to arrhythmias and other causes of cardiovascular death. ${ }^{10}$ The insignificant difference of SST2 levels in the group of patients with arrhythmia, cardiogenic shock, and sudden cardiac arrest in this study remained unexplained. However, the difference in the follow-up period between this study and other studies was expected to show different results. This study only assessed the occurrence of complications during hospitalization, a study by Aldous et al. followed-up for 18 months.

The subjects of the study were divided into two groups based on the outcome, into the group of patients who died $(n=4)$ and those who survived $(n=38)$. The Mann-Whitney test was performed to compare sST2 levels in the group of patients who died with patients who survived with the results summarized in Table 3.

Table 3. The difference in sST2 levels between death and survival group

\begin{tabular}{llcl}
\hline & \multicolumn{2}{c}{$\mathbf{s S T 2}(\mathbf{n g} / \mathbf{m L})$} & \multirow{2}{*}{ * $^{*}$} \\
\cline { 2 - 3 } & Mean & SD & \\
\hline Death & 164.05 & 77.35 & 0.027 \\
Survive & 72.55 & 73.15 & \\
\hline
\end{tabular}

*Mann-Whitney

The results of this study found that SST2 levels were significantly higher in the group of patients who died than the group of patients who survived $(p=0.027)$. A population-based study reported a twice-increased risk of death in the group with higher SST2 levels. ${ }^{24}$ Another study has shown that sST2 levels can be used to predict one-year mortality in NSTEMI patients. ${ }^{12}$

Table 4. Correlation of SST2 levels and length of stay

\begin{tabular}{ccc}
\hline & \multicolumn{2}{c}{$\mathbf{s S T 2}(\mathbf{n g} / \mathbf{m L})$} \\
\cline { 2 - 3 } & $\mathbf{r}$ & $\mathbf{p}^{*}$ \\
\hline Length of stay & -0.11 & 0.947 \\
\hline
\end{tabular}

*Spearman correlation test

Spearman test showed no correlation between sST2 levels and length of stay ( $p=0.947)$, as shown in Table 4. This result was probably because this study did not consider pharmacotherapy or intervention and other technical factors that might extend the length of stay. This study has several limitations, including no consideration of homogeneous onset 
of chest pain in the subjects,and SST2 reached its peak level at 6 to 17 hours after myocardial injury and will decrease to stable levels at 20-42 hours. ${ }^{11}$ The wide variety of the onset of chest pain causes the possibility of bias due to the difference in the time of measurement of sST2 levels. Another limitation was no analysis of intervention and therapy. The variety of treatments and interventions received by patients might affect the length of stay and outcome.

\section{CONCLUSION AND SUGGESTION}

sST2levels can be used as a prognostic marker for NSTEMI patients, especially in case of heart failure and patient outcomes. There was no statistical difference in SST2 levels between patients with complications of arrhythmias, cardiogenic shock, and sudden cardiac arrest compared to patients without complications. Further research considering the management of patients and the onset of chest pain for the inclusion criteria were needed to reduce bias to determine the correlation of SST2 correlation with length of stay and patient outcomes.

\section{REFERENCES}

1. Reed GW, Rossi JE, Cannon CP. Acute myocardial infarction. The Lancet, 2017; 389(10065): 197-210.

2. World Health Organization in collaboration with the World Heart Federation and the World Stroke Organization. Global Atlas on Cardiovascular Disease Prevention and Control, 2011; 8-13.

3. Mullasari AS, Balaji P, Khando T. Managing complications in acute myocardial infarction. J Assoc Physicians India, 2011; 59:43-8.

4. Bajaj A, Sethi A, Rathor P, Suppogu N, Sethi A. Acute complications of myocardial infarction in the current era: Diagnosis and management. Journal of Investigative Medicine, 2015; 63(7): 844-855.

5. Bahit MC, Lopes RD, Clare RM, Newby LK, PieperKS, Van de Werf $F$, et al. Heart failure complicating non-ST-segment elevation acute coronary syndrome: Timing, predictors, and clinical outcomes. JACC: Heart Failure, 2013; 1(3): 223-229.

6. Roubín S, Abu-Assi E, López-López A, Bouzas-Cruz N, Castiñeira-Busto M, Cambeiro-González, et al. Risk stratification for the development of heart failure after acute coronary syndrome at the time of hospital discharge: Predictive ability of GRACE risk score. Journal of Cardiology, 2015; 66(3): 224-231.

7. Salvagno GL, Pavan C. Prognostic biomarkers in acute coronary syndrome. Annals of Translational Medicine, 2016; 4(13): 1-8.

8. Demyanets S, Speid WS, Tentzeris I, Jarai R, Katsaros KM, Farhan S. Soluble ST2 and interleukin-33 levels in coronary artery disease: relation to disease activity and adverse outcome. PLoS One, 2014; 9(4): e95055.

9. Konukoglu D. Is soluble ST2 a new marker in heart failure?. International Journal of Medical Biochemistry, 2018; 1(1): 44-51.

10. Genis $A$, de Antonio $M$, Vila J, Peñafiel J, Galán A, Barallat J, et al. Head-to-head comparison of 2 myocardial fibrosis biomarkers for long-term heart failure risk stratification: ST2 versus galectin-3. Journal of the American College of Cardiology, 2014; 63(2): 158-166.

11. Richards AM, Di Somma S, Mueller T. ST2 in stable and unstable ischemic heart diseases. The American Journal of Cardiology, 2015; 115(7): 48B-58B.

12. Eggers KM, Armstrong PW, Califf RM, Simoons ML, Venge P, Wallentin L, James SK. ST2 and mortality in non-ST-segment elevation acute coronary syndrome. American Heart Journal, 2010; 159(5): 788-794.

13. Barbarash O, Gruzdeva O, Uchasova E, Dyleva $Y$, Belik E, Akbasheva O, et al. Prognostic value of soluble ST2 during hospitalization for ST-segment elevation myocardial infarction. Annals of Laboratory Medicine, 2016; 36(4): 313-319.

14. Aldous SJ, Richards AM, Troughton R, Than M. ST2 has diagnostic and prognostic utility for all-cause mortality and heart failure in patients presenting to the emergency department with chest pain. Journal of Cardiac Failure, 2012; 18(4): 304-310.

15. Gomar F, Perez-Quilis C, Leischik R, Lucia A. Epidemiology of coronary heart disease and acute coronary syndrome. Annals of Translational Medicine, 2016; 4(13): 256.

16. Kytö V, Sipilä J, Rautava P. Association of age and gender with risk for non-ST-elevation myocardial infarction. European Journal of Preventive Cardiology, 2015; 22(8): 1003-1008.

17. Hamm CW, Heeschen C, Falk E, Fox KA. Acute coronary syndromes: Pathophysiology, Diagnosis, and Risk Stratification. 2014; 333-365.

18. Lapu-Bula R, Onwuanyi A, Bielo MV, Deffer O, Quarshie A, Alema-Mensah E, et al. Risk factors for acute non-ST-segment elevation myocardial infarction in a population sample of predominantly African American patients with chest pain and normal coronary arteries. Ethnicity \&Disease, 2011; 21(4): 421.

19. Hubacek JA, Stanek V, Gebauerova M, Adamkova V, Lesauskaite $V$, Zaliaduonyte-Peksiene $\mathrm{D}$, et al. Traditional risk factors of acute coronary syndrome in four different male populations-total cholesterol value does not seem to be relevant risk factor. Physiol Res, 2017; 66(1): S121-S128.

20. Kohli P, Bonaca MP, Kakkar R, Kudinova AY, Scirica BM, Sabatine MS, et al. Role of ST2 in non-ST-elevation acute coronary syndrome in the MERLIN-TIMI 36 trial. Clin Chem, 2012; 58(1): 257e266.

21. Bhardwaj A and Januzzi JL. ST2: A novel biomarker for heart failure. Expert Review of Molecular Diagnostics, 2010; 10(4): 459-464.

22. Hashimoto T, Kass DA. Stressed hearts inflame the body (in a good way). Proceedings of the National 
Academy of Sciences. 2015; 112(23): 7113-7114.

23. Ciccone MM, Cortese F, Gesuald M, Riccardi R, Di Nunzio D, Moncelli $M$, et al. A novel cardiac bio-marker: ST2: a review. Molecules, 2013; 18(12): 15314-15328.
24. Chen LQ, de Lemos JA, Das SR, Ayers CR, Rohatgi A. Soluble ST2 is associated with all-cause and cardiovascular mortality in a population-based cohort: The Dallas Heart Study. Clinical Chemistry, 2013; 59(3): 536-546. 